\title{
The rotational motion simulation of AIST small spacecraft prototype based on current values from solar battery panels and on a hardware- software stand
}

\author{
Andry Sedelnikov ${ }^{1}$, Sergey Safronov ${ }^{1}$, Ekaterina Khnyryova $^{2}$ \\ ${ }^{1}$ Department of Space Engineering, Samara National Research University, 34, Moskovskoye shosse, \\ Samara, 443086, Russia \\ ${ }^{2}$ Department of Further Mathematics, Samara National Research University, 34, Moskovskoye shosse, \\ Samara, 443086, Russia
}

Received: July 2, 2021. Revised: November 15, 2021. Accepted: December 9, 2021. Published: December 29, 2021.

\begin{abstract}
The functioning of space technique is associated with remote maintenance of operable state of onboard systems and software. If an element fails, problems arise in analyzing the state, ascertaining the reasons for the failure and restoring the element functional using available hardware-in-the-loop and algorithmic tools. The paper concentrates on currents analysis from solar battery panels of AIST small spacecraft in order to evaluate the parameters of the satellite's rotational motion after a significant break-down of the electrical battery. At the same time, the scientific equipment and onboard measurement instruments proved to be practically inoperative due to the lack of power supply. After the break-down of the electrical battery, magnetorquers and measurement instruments could not perform their function. A backup orientation system was not provided. The raw data for estimating the angular velocity vector was the current values from solar battery panels. However, in order to obtain an acceptable estimate of the angular velocity vector, more accurate current measurements are required than it implemented onboard the small spacecraft. To simulate the small spacecraft rotational motion and compare results with estimates obtained from telemetric data analysis, HIL US-03 hardware-software stand for simulation of the small spacecraft systems was used.
\end{abstract}

Keywords - data measurement analysis, magnetometer, microacceleration, small spacecraft.

\section{INTRODUCTION}

I $\mathrm{N}$ the small spacecraft operation various abnormal situations can arise related to break-down of the main measurement instruments. The paper [1] describes the changing of small spacecraft for communications to a state of uncontrolled rotation after an abnormal situation. In its telemetric data, there were no data about its rotation motion parameters and angular momentum of reaction wheels, which were used as magnetorquers of pointing and control system. Using data on current from solar battery panels, the author of [1] established that the angular velocity module of small spacecraft rotation was within $(0.6-0.75) \pm 0.02 \mathrm{deg} / \mathrm{s}$. It allowed making a timely decision to unload the angular momentum of reaction wheels.

In the flight tests of the Munin nanosatellite, the magnetometer failure resulted in a situation where more exact data on the small spacecraft evolution around the centre of mass was data about the current collecting from the solar battery [2].

Thus, using the data on the current is relevant in order to attempt to control the small spacecraft rotational motion with its degraded performance when the standard measurement instruments are failure.

The conditions occurs when the small spacecraft has worked off the planned period of active existence and the electrical battery has broken-down. As a result, practically all the target and supporting equipment is inoperative. However, telemetering measurements arrive, and they contain the data on current from solar battery panels. Such situation is typical 
for the flight model and prototype of AIST small spacecraft [3-5]. These small spacecrafts were operated in an uncontrolled flight. Therefore, data on the current can be use not as raw data for the effective operation of pointing and control system, but as raw data for estimating the parameters of the small spacecraft rotational motion. The analysing the small spacecraft rotational motion under conditions of prolonged exposure of perturbations in the absence of control actions is of great interest.

\section{THE ACCURACY COMPARISON OF ANGULAR VELOCITY ESTIMATION OF SMALL SPACECRAFT ROTATION WITH ESTIMATIONS BY OTHER MEASUREMENT INSTRUMENTS}

In the AIST small spacecraft prototype before the breakdown of the electrical battery, the basic means for estimating the parameters of the small spacecraft rotational motion around the centre of mass were the magnetometer sensors. Figure 1 presents the comparison results of angular velocity estimations by means magnetometer sensors and the data on current from solar battery panels obtained on 09 September 2014.
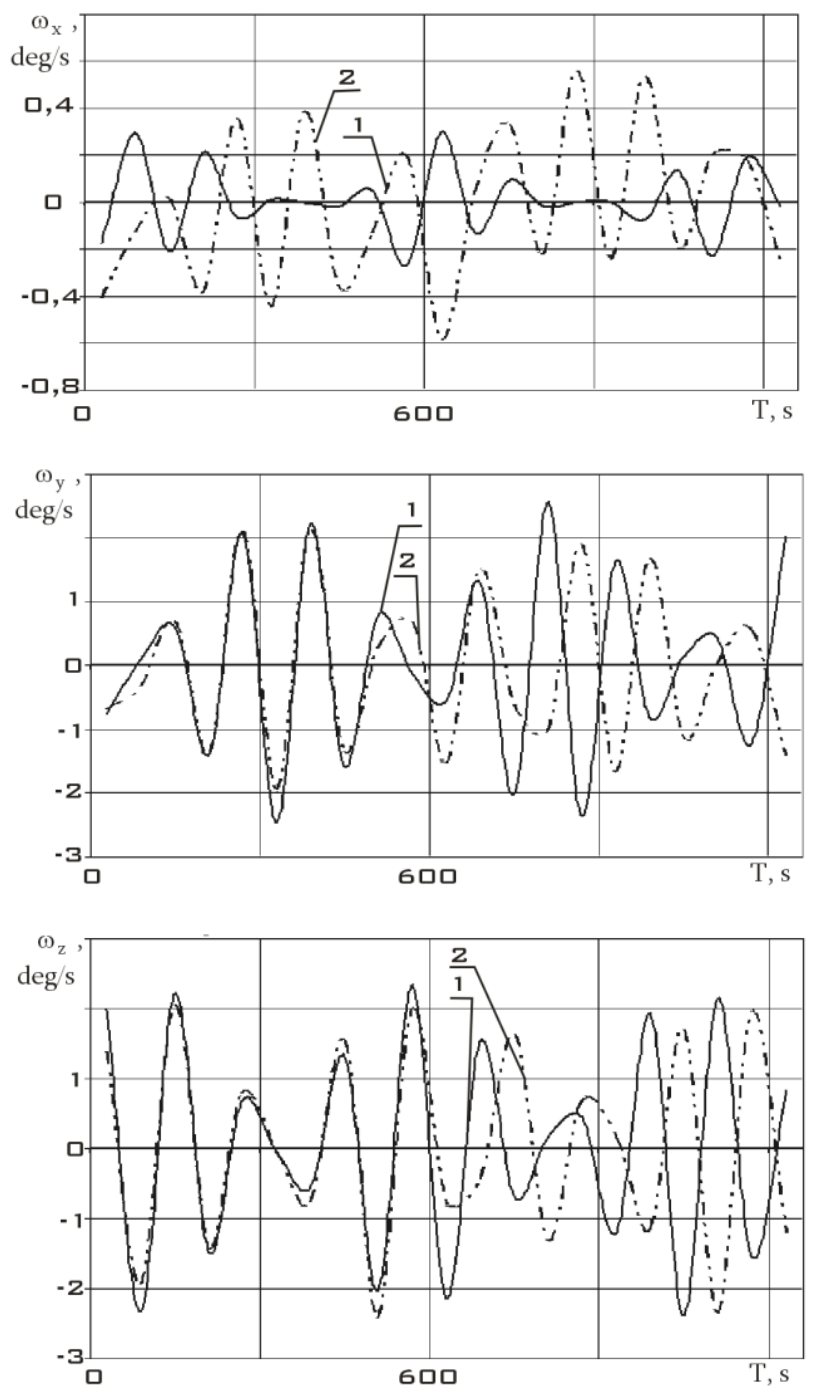

Fig. 1. Components estimations of angular velocity of AIST small spacecraft's prototype rotation by means magnetometer sensors (2) and the data on current from solar battery panels

The angular velocity estimation of AIST small spacecraft rotation based on the measurements of the Earth's magnetic field induction vector was carried out according to the following formulae [6]:

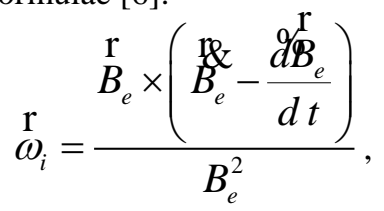

where $\vec{\omega}_{i}$ is angular velocity vector of small spacecraft rotation of the around the center of mass at $t_{i}$ time moment; $\stackrel{1}{B}_{e}$ is Earth's magnetic field induction vector; $\frac{d \mathbb{B}_{e}}{d t}$ is local derivative of the Earth's magnetic field induction vector in the body axes coordinate system, which is due mainly to the small spacecraft motion around the center of mass; $B_{e}$ is the absolute rate of change the Earth's magnetic field induction vector, which is due mainly to the small spacecraft motion of the center of mass.

The angular velocity estimation of AIST small spacecraft rotation based on the data on current from solar battery panels was carried out according to the following formulae [7]:

$$
\omega_{k i}=\frac{\arccos \left(\frac{i_{k i}}{i_{\text {max }}}\right)-\arccos \left(\frac{i_{k i-1}}{i_{\text {max }}}\right)}{t_{i}-t_{i-1}},
$$

where $i_{k i}$ and $i_{k i-1}$ are measured values of the current intensity at $t_{i}$ and $t_{i-1}$ times moment; $i_{\max }$ is the maximum value of the current intensity, which is considered invariable and the same for all AIST small spacecraft's facets; $k=x, y, z$.

The correspondence analysis on Figure 1 shows that significant differences in the given time interval are observed only for the angular velocity component $\left(\omega_{x}\right)$. However, it is noted in [3] that the correspondence of the magnetometer sensors at this measurement channel was unsatisfactory. Therefore, referring to the investigation [3], we can assume that in general, the numerical characteristics of the angular velocity (mathematical expectation and variance) are correctly estimated using data on the current from solar battery panels.

\section{ESTIMATION OF SMALL SPACECRAFT'S ROTATIONAL MOTION PARAMETERS USING THE DATA ON CURRENT FROM SOLAR BATTERY PANELS}

Let us consider the angular velocity estimation of rotation of AIST small spacecraft prototype when the target and supporting equipment did not operate due to the break-down of the electrical battery. Figure 2 shows the dependences of the angular velocity modulus estimated from the data on current from solar battery panels obtained on 01 June 2018 . 


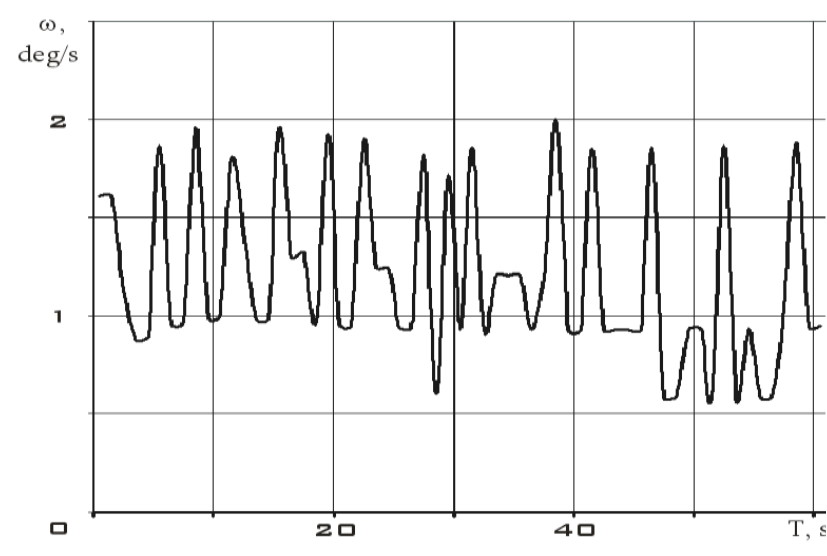

Fig. 2. The angular velocity module's Estimates of the AIST small spacecraft's prototype rotation using the data on current from solar battery panels on 1 June 2018.

The analysis of these dependences show, that:

1.the small spacecraft gyrodynamics has not changed significantly since the operation end of the standard measurement instruments.

2.the angular velocity dependence on time contains significant harmonics up to a frequency of 3 radians per second. Therefore, the angular velocity estimation by standard measurement instruments could be carried out with limited accuracy (the data output frequency via the telemetering channel is 1 time in 6 seconds).

3.for effective control of the angular velocity, it is necessary to increase the data output frequency and the accuracy of measuring the electrical battery charging current.

Unfortunately, increasing the data output frequency and accuracy is impossible now on the flight model and prototype of AIST small spacecraft. Such updating was not envisaged at first. Software reboot is also impossible when the electrical battery has broken-down. However, it is necessary to take into account the operational experience of the flight model and prototype of AIST small spacecraft for the improving the angular velocity damping system. The application of the improved system is assumed on the following small spacecraft models of AIST series. Their launches are planned for 2023. In this situation, in order to simulate correctly the rotational motion of the flight model and prototype of AIST small spacecraft, it is necessary to use additionally the simulation model.

\section{THE SIMULATION MODEL DEVELOPMENT OF THE SMALL SPACECRAFT ROTATIONAL MOVEMENT}

As a simulation model in this study, we use the HIL US03 hardware-software stand for simulation of small spacecraft systems (figure 3).

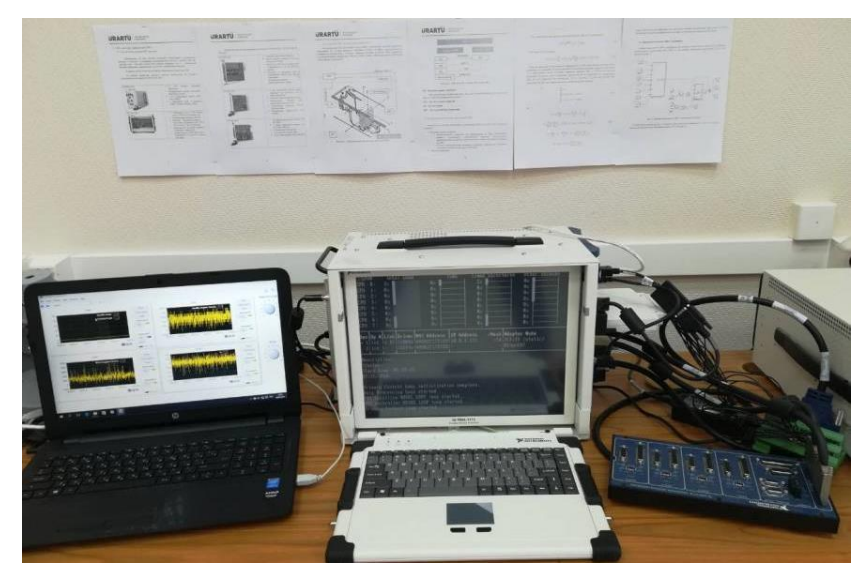

Fig. 3. The HIL US-03 hardware-software stand for simulation of small spacecraft systems.

Hardware-software simulation is a method that is used in the development and testing of real-time systems, which can include any system of small spacecraft, as well as the onboard control system.

Using the hardware-software stand, we plan to simulate the rotational motion of the small spacecraft model in a magnetic field similar to the field that is affecting on the small spacecraft in the working orbit. In addition, we need a solar radiation simulator.

The small spacecraft model should meet the requirements of the research tasks. In particular, it should have in its composition magnetometers, as well as the solar battery elements, placed on each of the six facets of the model. The model must be a cube.

To implement hardware-software simulation of rotational motion, it is necessary:

- to develop a conceptual model of a system for analyzing the measurements of magnetometers and currents from solar battery elements based on analysis of typical processes, structures and hardware blocks;

- to develop algorithms and approaches for the conceptual model implementation on the software and hardware of simulating complex;

- to develop the system structure for analyzing the measurements of magnetometers and currents from solar battery elements. The system for analyzing includes hardware (magnetometers, solar battery elements, magnet coils, solar simulators, etc.) and software that provide the hardware operation control and simulate the orbital flight conditions (external environment), disturbances and so on;

- to adjust the simulating complex to the corresponding structure of the measurement analysis system programmatically by means of the programming possibility the controller structure;

- to develop software for controlling the hardware operation;

- to develop software that simulate the orbital flight conditions;

- to test the system for analyzing measurements by multiple launching the simulation stand in different modes. With satisfactory performance of the computing instruments 
of the complex, the system study process can be carried out in real time.

The HIL system consists of three main components (figure 4):

- real-time processor;

- input-output interfaces;

- operator interface.

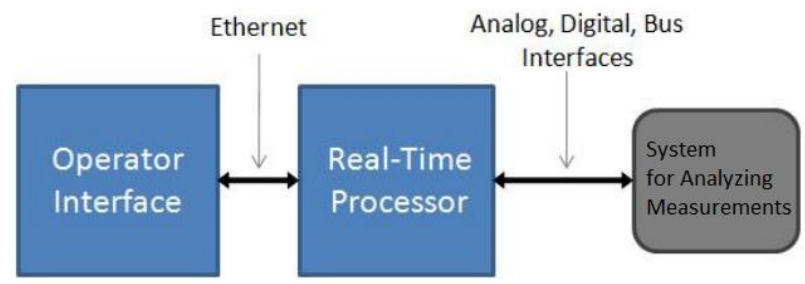

Fig. 4. The hardware-software stand for simulation of small spacecraft systems

Software-hardware simulating allows improving the testing quality, reducing the impact of the human factor, reducing the time and the development cost.

The results of studied system's analysis are recommendations for adjusting the system (requirements for the data output frequency and accuracy of current measurement) and algorithms for estimating the angular velocities about the body axes of the small spacecraft model. They will be used for developing the backup system for determining the small spacecraft orientation.

Special hardware and software onboard any small spacecraft allow in the event of a failure to restore the satellite's functionality due to redistribution of functions and tasks between operable devices and onboard equipment modules [8]. At the same time, the further meeting of the small spacecraft targets is ensured, and the reliability and survivability of the small spacecraft are increased.

\section{References}

[1] A.A. Davydov, "Determination of parameters of attitude motion of the spacecraft from the telemetry data on solar battery current", Cosmic Research, vol. 49, 2011, pp. 345-354.

[2] M.Yu. Ovchinnikov, V.I. Penkov, D.S. Roldugin and D.S. Ivanov, "Magnetic systems for small spacecraft orientation", Keldysh Institute of Applied Mathematics Russian Academy of Sciences, 2016.

[3] T.A. Ivashova, "The use of measuring current information from solar panels to estimate the angular velocity of rotation of a small spacecraft", in Proc. 2020 International Multi-Conference on Industrial Engineering and Modern Technologies (FarEastCon), Vladivostok, Russia, 2020, pp. 1-6.

[4] A.S. Gorozhankina, D.I. Orlov and D.A. Belousova, "Problems of development motion control algorithms for a small spacecraft for technological purpose taking into account temperature deformations of solar panels", Journal of Physics: Conference Series, vol. 1546, 2020, 012015 .
[5] A.V. Sedelnikov, A.S. Filippov and A.S. Gorozhakina, "Evaluation of calibration accuracy of magnetometer sensors of AIST small spacecraft", Journal of Physics: Conference Series, vol. 1015, 2018, 032045.

[6] A.V. Sedelnikov, "Fractal quality of microaccelerations", Microgravity Science and Technology, vol. 24, 2012, pp. 345-350.

[7] D.I. Orlov, "Modeling the temperature shock impact on the movement of a small technological spacecraft", in Proc. International Conference "Problems of Applied Mechanics", Bryansk, Russia, 1-3 December 2020, 050001.

[8] R. N. Akhmetov, V. P. Makarov and A. V. Sollogub, "Bypass principles of automated spacecraft survivability control", in Proc. ICINS 2016, Saint Petersburg, Russia, 2016, pp. 501-505.

\section{Creative Commons Attribution License 4.0 (Attribution 4.0 International, CC BY 4.0)}

This article is published under the terms of the Creative Commons Attribution License 4.0 https://creativecommons.org/licenses/by/4.0/deed.en_US 